\title{
Mechanical occlusion of the left atrial appendage - lessons from surgical experience
}

\section{Dawn S. Hui and Richard Lee}

We read with interest the Review by Caliskan et al. on left atrial appendage (LAA) occlusion (Caliskan, E. et al. Interventional and surgical occlusion of the left atrial appendage. Nat. Rev. Cardiol. 14, 727-743; 2017) ${ }^{1}$. We appreciate that a broad review of this clinical problem is important; however, a critical, and not merely comprehensive, review is needed to fully appreciate the problem of LAA occlusion - namely, the failure of any single technique to reliably and completely occlude the LAA. Caliskan and colleagues' review of technologies would have been better informed by a discussion of the underlying reasons for the failure of these techniques.

LAA anatomy and function are complex. To achieve the ultimate goal of stroke prevention, occlusion must completely smooth the left atrial endocardial surface. One anatomical consideration is the proximity of the circumflex artery to the LAA base. With all occlusion approaches, avoidance of coronary injury might preclude complete occlusion. Furthermore, external devices must navigate the anatomical relationship of the external base to the internal orientation of the appendage ostium, which might also prohibit complete coaptation, leaving a persistent stump. With increasing stand-alone maze procedure volumes, the majority being off-pump ${ }^{2}$, externally applied devices will remain an important technique. From the extensive discussion in their Review on the AtriClip study ${ }^{3}$ conducted by Caliskan and colleagues, one might understand that confidence in this device is assured. We would caution against reaching this conclusion before knowing the limitations of that study. First, the definition of 'success' in this study allowed for a residual stump of up to $10 \mathrm{~mm}$. Although this definition is used in other studies ${ }^{4-6}$, it is arbitrary, and indeed we have reported a case of LAA thrombus in a residual stump of $<10 \mathrm{~mm}$ despite fully therapeutic anticoagulation ${ }^{7}$. Second, the only transoesophageal echocardiography (TEE) assessment in the AtriClip study was intraoperative; mid-term assessment was performed in only $7.9 \%$ of the cohort with the use of CT imaging. Finally, relative stroke risk reduction was based on the $\mathrm{CHA}_{2} \mathrm{DS}_{2}$ VASc score, not a control group. Although the $\mathrm{CHA}_{2} \mathrm{DS}_{2}$-VASc score has a C-statistic of $0.8-0.9$, this score is not a perfect predictor ${ }^{8}$.

Another surgical approach is from within the LAA, allowing direct visualization and manipulation of the endocardial surface. Theoretically, this strategy should offer a greater chance of success. The challenge lies in the morphological LAA changes that occur when transitioning from an arrested, empty heart to the full, contracting state. Caliskan et al. cite several, old, retrospective studies utilizing internal ligation, but neglect contemporary papers. In our prospective, randomized study of three primary surgical techniques ${ }^{5}$, we found that no single technique was superior. The important lesson was that types of failure varied by technique. In the case of internal ligation, residual gaps and internal flow communications developed, probably owing to tension on the internal suture over time ${ }^{9}$. The contribution of atrial size and contractility is not yet known.
These data support the need for definitive LAA assessment before making decisions on anticoagulation cessation. This decisionmaking requires dedicated imaging. In our practice, we have found challenges in obtaining postoperative TEE, as guidelines do not address the substantial failure rates of LAA closure. Similar to the related clinical problem of atrial fibrillation, assessment of LAA occlusion should consist of more than a single intraoperative evaluation. Continued work on surgical techniques, with an eye towards long-term success, is important, but so is an honest evaluation of the limitations of current techniques and definitions of success.

Dawn S. Hui and Richard Lee are at the Center for Comprehensive Cardiovascular Care, Saint Louis University, Saint Louis, MO, USA.

Correspondence to R.L. rick.lee@health.slu.edu

doi:10.1038/nrcardio.2018.7 Published online 13 Feb 2018

1. Caliskan, E. et al. Interventional and surgical occlusion of the left atrial appendage. Nat. Rev. Cardiol. 14, 727-743 (2017)

2. Ad, N. et al. Surgical ablation of atrial fibrillation trends and outcomes in North America. J. Thorac Cardiovasc. Surg. 144, 1051-1060 (2012).

3. Caliskan, E. et al. Epicardial left atrial appendage AtriClip occlusion reduces the incidence of stroke in patients with atrial fibrillation undergoing cardiac surgery. Europace https://doi.org/10.1093/europace/ eux211 (2017)

4. Kanderian, A. S. et al. Success of surgical left atrial appendage closure: assessment by transesophageal echocardiography. J. Am. Coll. Cardiol. 52, 924-929 (2008).

5. Lee, R. et al. A randomized, prospective pilot comparison of 3 atrial appendage elimination techniques: Internal ligation, stapled excision, and surgical excision. J. Thorac. Cardiovasc. Surg. 152, 1075-1080 (2016).

6. Ward, A. F. et al. Totally endoscopic robotic left atrial appendage closure demonstrates high success rate. Innovations 12, 46-49 (2017).

7. Hui, D. S. et al. Left atrial appendage thrombus after successful surgical exclusion on anticoagulation: a need for closer postintervention monitoring. Ann. Thorac. Surg. 98, 1478 (2014).

8. Olesen, J. B. et al. Validation of risk stratification schemes for predicting stroke and thromboembolism in patients with atrial fibrillation: nationwide cohort study. BMJ 342, d124 (2011).

9. Hui, D. S. \& Lee, R. Modified internal ligation of the left atrial appendage. J. Thorac. Cardiovasc. Surg. 154, 847-848 (2017).

Competing interests

The authors declare no competing interests. 